\title{
Nodal Volume as a Prognostic Factor in Oral Tongue Cancer with Cervical Lymph Node Metastasis
}

\author{
Rattawut Wiengnon ${ }^{1}$, worawat rawangban ${ }^{2}$, Chakkrapong Chakkabatr ${ }^{1}$, and Napadon \\ Tangjaturonrasme ${ }^{3}$ \\ ${ }^{1}$ King Chulalongkorn Memorial Hospital \\ ${ }^{2}$ chulalongkorn university \\ ${ }^{3}$ Chulalongkorn University
}

May 26, 2021

\begin{abstract}
Objectives Oral tongue cancer is the most prevalent type of oral cavity cancer and presents the worst prognosis. With the use of tumor, lymph node, distant metastasis (TNM) staging system, only the tumor and lymph node size are taken into account. However, several studies have considered the tumor volume as a possible significant prognostic factor of oral tongue cancer with cervical lymph node metastasis. Our study, therefore, aimed to explore the prognostic implications of the relevant nodal volume. Design, Setting and Participants Medical records and imaging (either from computed tomography scan, CT scan or magnetic resonance imaging scan, MRI scan) of 70 patients diagnosed with oral tongue cancer with cervical lymph node metastasis between January 2011 and December 2016 were retrospectively reviewed. Main outcome measures The nodal volume was measured from the imaging using the Eclipse application (Version 15.6.05, Varian company) and was further analyzed for its prognostic implications, particularly on overall survival, disease-free survival, and distant metastasis-free survival. Results From a receiver operating characteristic (ROC) curve analysis, the optimal cut-off value of the nodal volume was $3.95 \mathrm{~cm} 3$, to predict the disease prognosis, in terms of overall survival and metastatic-free survival $(\mathrm{p}=<0.001$ and $\mathrm{p}=0.005$, respectively), but not the disease-free survival $(\mathrm{p}=0.241)$. For the multivariable analysis, the nodal volume, but not TNM staging, was a significant prognostic factor for distant metastasis $(\mathrm{HR}=4.5,95 \% \mathrm{CI} 1.1-17.94, \mathrm{p}=0.036 \mathrm{vs} \mathrm{p}=0.459$, respectively). Conclusions In patients with oral tongue cancer and cervical lymph node metastasis, the presence of a nodal volume of $3.95 \mathrm{~cm} 3$ was a poor prognostic factor for distant metastasis. Therefore, the lymph node volume from the current imaging protocol may be useful in adjunct with the current staging system to predict the disease prognosis
\end{abstract}

\section{Nodal Volume as a Prognostic Factor in}

\section{Oral Tongue Cancer with Cervical Lymph Node Metastasis}

\section{Abstract}

\section{Objectives}

Oral tongue cancer is the most prevalent type of oral cavity cancer and presents the worst prognosis. With the use of tumor, lymph node, distant metastasis (TNM) staging system, only the tumor and lymph node size are taken into account. However, several studies have considered the tumor volume as a possible significant prognostic factor. Our study, therefore, aimed to explore the prognostic implications of the relevant nodal volume.

\section{Design, Setting and Participants}


Medical records and imaging (either from CT or MRI scan) of 70 patients diagnosed with oral tongue cancer with cervical lymph node metastasis between January 2011 and December 2016 were retrospectively reviewed.

\section{Main outcome measures}

The nodal volume was measured from the imaging using the Eclipse application and was further analyzed for its prognostic implications, particularly on overall survival, disease-free survival, and distant metastasis-free survival.

\section{Results}

From ROC curve analysis, the optimal cut-off value of the nodal volume was $3.95 \mathrm{~cm}^{3}$, to predict the disease prognosis, in terms of overall survival and metastatic-free survival ( $\mathrm{p}=<0.001$ and $\mathrm{p}=0.005$, respectively), but not the disease-free survival $(\mathrm{p}=0.241)$. For the multivariable analysis, the nodal volume, but not TNM staging, was a significant prognostic factor for distant metastasis

\section{Conclusions}

In patients with oral tongue cancer and cervical lymph node metastasis, the presence of a nodal volume of [?] $3.95 \mathrm{~cm}^{3}$ was a poor prognostic factor for distant metastasis. Therefore, the lymph node volume may be useful in adjunct with the current staging system to predict the disease prognosis.

\section{Key points}

- In general, TNM staging system, which the volume of tumor is not included in the system, has been used to predict the disease prognosis in oral cavity cancer

- Recent studies, the tumor volume has increasing evidence for its possible prognostic implication

- We conducted a retrospective cohort study investigating association between metastic cervical lymph node volume in oral tongue cancer and its prognostic implication

- The result shows presence of a nodal volume of [?]3.95 $\mathrm{cm}^{3}$ was a poor prognostic factor for distant metastasis

- The lymph node volume may be useful in adjunct with the TNM staging system to predict the disease prognosis

\section{Objectives}

In Thailand, the cancer mortality rate had been increasing in numbers over the years. (1) Among them, oral cavity cancer is the seventh most common cancer in Thai male, and the tongue is the most common site. The incidence of oral tongue cancer is 2.2 and 1.0 per 100,000 males and females, respectively. Overall, the 5 -year survival rate of oral cavity cancer is $25.9 \%$, where tongue cancer has the worst prognosis. (3)

In general, the tumor, lymph node, distant metastasis (TNM) staging system has been used to predict the disease prognosis, where size, number, and location of the tumor $(\mathrm{T})$ and lymph node $(\mathrm{N})$ and the presence of distant metastasis (M) are the primary considerations. However, in recent studies, the tumor volume has increasing evidence for its possible prognostic implication. Particularly in head and neck cancer, the tumor volume is a promising prognostic factor for predicting disease recurrence and distance metastasis. (4-5)

Therefore, our study aims to explore the use of relevant nodal volume on its prognostic implication and the possible application of nodal volume in addition to the current staging system.

\section{Design, Setting and Participants}

This retrospective cohort study was approved by the Research Ethics Committee, Faculty of Medicine, Chulalongkorn University. The protocol was performed in accordance with the declaration of Helsinki and 
its later amendments. Informed consent was waived due to an anonymous data extraction with no direct patient and public involvement in the study.

\section{Study participants}

Medical records of patients diagnosed with oral tongue cancer and cervical lymph node metastasis between January 2011 and December 2016 were reviewed. The inclusion criteria were as follows: 1) diagnosed with oral tongues cancer with cervical lymph node metastasis between January 2011 and December 2016; 2) had the pre-operative CT or MRI scans compatible with the Eclipse application (Version 15.6.05, Varian company); and 3) had the characteristics of lymph node metastasis including short-axis diameter of $>1 \mathrm{~cm}$, necrotic/cystic content, irregular border, and matted lymph node. The study excluded patients younger than 18 years old or non-Thais.

\section{Main outcome measures}

\section{Measures of lymph node volume}

In every slice of the CT or MRI scan imaging, the border of the lymph node was manually drawn using the Eclipse application (by the RC under the supervision of $\mathrm{CC}$ ) to create the three-dimensional image. The nodal volume was then measured. All cervical lymph nodes were measured to represent the nodal volume of the patient. (Figure 1)

\section{Data collection}

All demographics and clinical characteristic data of the patients, including sex, age, stage of the disease, the nodal volume, the date of disease diagnosis, the date of metastasis diagnosis, and the date of the death, were collected.

\section{Statistical analysis}

All statistical analysis was performed using STATA version 14.2. The Kolmogorov-

Smirnov test was used to evaluate the probability distribution of the samples with the reference data. Categorical data were described as median and percentages. A receiver operating characteristic (ROC) curve analysis was performed to identify the nodal volume's optimal cut-off value with the highest possible sensitivity and specificity. For survival analysis, the Kaplan-Meier method and the log-rank test were used. The Cox proportional hazard model and the univariate and multivariate analysis were performed to evaluate the prognostic factors. Overall survival, disease-free survival, and distant metastasis-free survival were considered to determine the prognostic implications. The p-value of $<0.05$ was considered statistically significant.

\section{Results}

Overall, 236 patients were diagnosed with oral tongue cancer between January 2011 and December 2016; however, 45 patients had no pre-operative CT or MRI scan imaging, 118 patients had no pathologic lymph node, and three patients were non-Thais. Finally, 70 patients were included in the study.

Males were predominant (67.1\%). The mean age (SD) was 58 (SD) years old. $62.8 \%$ of the patients were in stage IVA, and $70 \%$ of the histopathological results were well-differentiated squamous cell carcinoma (SCCA). For overall survival analysis, the patients' median survival time was 13 months, the 1-year survival rate was $51 \%$, and the 3 -year survival rate was $24 \%$.

Recurrence of the disease was found in 17 patients (24.2\%), which the sites of recurrence were oral tongue (14 patients) and cervical lymph node (3 patients). The distant metastasis was present in 19 patients, including lungs (12 patients), bones (4 patients), and other sites (3 patients).

The mean (SD) nodal volume was $5.31(\mathrm{SD}) \mathrm{cm}^{3}$. In ROC curve analysis, the optimal cut-off value for the nodal volume was $3.95 \mathrm{~cm}^{3}$ (Area under the curve $($ AUC) $=0.672$, Sensitivity $=65.5 \%$, and Specificity $=$ $73.3 \%$ ) (Figure 2). Regarding the optimal nodal volume cut-off value, the patients were classified into 1) 
the nodal volume of $<3.95 \mathrm{~cm}^{3}$ group (30/70 patients, $\left.42.9 \%\right)$ and 2) the nodal volume of [?]3.95 $\mathrm{cm}^{3}$ group (40/70 patients, $57.1 \%$ ). The patients' demographics and clinical characteristics are illustrated in Table 1.

Additionally, the median (IQR) nodal volume of the patients in the dead group was significantly higher than the alive group $\left(6.5 \mathrm{~cm}^{3}(2.49-23.12)\right.$ vs $\left.3.1 \mathrm{~cm}^{3}(1.9-7.7), \mathrm{p}=0.043\right)$. The nodal volume of the patients with distant metastasis was likely to be larger than the patients with no metastasis. (Table 2)

\section{Survival outcome analysis}

The Kaplan-Meier survival curve of both groups, including the patients with the nodal volume of $<3.95$ $\mathrm{cm}^{3}$ and [?]3.95 $\mathrm{cm}^{3}$, were identified and compared using the log-rank test. Overall survival and distant metastasis-free survival were significantly different between the nodal volume of $<3.95 \mathrm{~cm}^{3}$ and $[?] 3.95 \mathrm{~cm}^{3}$ groups $(\mathrm{p}<0.001$ and $\mathrm{p}=0.005$, respectively), but not for the disease-free survival $(\mathrm{p}=0.241)$. (Figure 3 )

From 2-year survival analysis, overall survival rate of patients with the nodal volume of of $<3.95 \mathrm{~cm}^{3}$ and [?] $3.95 \mathrm{~cm}^{3}$ were $50 \%(95 \% \mathrm{CI}=0.3130-0.6612)$ and $12.5 \%(95 \% \mathrm{CI}=0.0458-0.2461)$, respectively. The metastasis-free survival rate of the nodal volume of $<3.95 \mathrm{~cm}^{3}$ and [?] $3.95 \mathrm{~cm}^{3}$ groups were $90 \%(95 \% \mathrm{CI}$ $=0.6707-0.9763)$ and $48 \%(95 \% \mathrm{CI}=0.2359-0.6978)$, respectively, while for the recurrent-free survival rate were $82.5 \%(95 \% \mathrm{CI}=0.5952-0.9311)$ and $55.49 \%(95 \% \mathrm{CI}=0.2426-0.7827)$, respectively.

\section{Multivariate analysis}

In univariate and multivariate analysis, the Cox proportional hazard model was applied to assess the association between the variables (e.g. age, sex, T stage, N stage, M stage, TNM stage, treatment options (with or without surgery), and nodal volume $\left(<3.95 \mathrm{~cm}^{3}\right.$ and $\left.[?] 3.95 \mathrm{~cm}^{3}\right)$ ), and the survival outcomes (e.g. overall survival, disease-free survival and distant metastasis-free survival). Only the nodal volume was significantly associated with the distant metastasis-free survival (Adj $\mathrm{HR}=4.45, \mathrm{p}=0.036,95 \% \mathrm{CI}=1.1-17.94$ ). The nodal volume was also likely to be associated with overall survival but not statistically significant. (Table 3 )

\section{Discussion}

In this study, the prognostic implications of the volume of the lymph node, or the nodal volume, in oral tongue cancer were described for the first time. The 1-year survival rate in our study was $51 \%$, which was slightly lower than the 1-year survival rate of oral cavity cancer stage III and IV reported by the National Cancer Intelligence Network (NCIN) (10). The difference could be partially explained by the different population and ethnicity.

From the ROC curve analysis, the optimal cut-off level of the nodal volume of $3.95 \mathrm{~cm}^{3}$ illustrated the diagnostic capability of the nodal volume to determine the survival outcomes, including overall survival and distant metastasis-free survival (sensitivity $=65.5 \%$, and specificity $=73.3 \%$ ). In multivariate analysis, only the nodal volume, but not the TNM stage, was a prognostic factor for distant metastasis-free survival (Adj $\mathrm{HR}=4.45, \mathrm{p}=0.036,95 \% \mathrm{CI}=1.1-17.94)$.

In general, the TNM staging system is used to predict the disease prognosis. The staging system mainly focuses on the size of the primary tumor and its invasion to the adjacent organs. Apart from the tumor size, the role of the tumor volume, or the overall volume of the primary tumor, predicting the disease prognosis has been reported with increasing evidence. Strongin A et al. indicated the tumor volume of $>35 \mathrm{~cm}^{3}$, but not the TNM stage was associated with the poor prognosis for recurrence and distant metastasis. (4) Lin CS et al. also revealed that the tumor volume was associated with the recurrent rate and the death rate. (6)

In our study, the role of the nodal volume on its prognostic implications was explored and demonstrated the significant association between the nodal volume and the disease prognosis. The study emphasized the superiority of the nodal volume over the TNM stage on predicting the disease prognosis, particularly the distant metastasis-free survival.

In multivariate analysis, the larger nodal volume (optimal cut-off volume $=3.95 \mathrm{~cm}^{3}$ ) was significantly 
associated with the likelihood of distant metastasis. The association between the nodal volume and the overall survival was also likely, but not statistically significant. (Adj $\mathrm{HR}=1.8, \mathrm{p}=0.062,95 \% \mathrm{CI}=0.97$ 3.34) The non-significance may be partly due to the small number of patients. With the smaller nodal volume, the $\mathrm{N}$ stage was likely to be lesser, andvice versa. Therefore, the patients in the groups classified by the nodal volume were not evenly distributed in terms of the $\mathrm{N}$ stage, thus affecting the statistical analysis.

In our study, the nodal volume revealed its prognostic implications over the TNM stage in terms of distant metastasis. This finding reflects the possible role of the nodal volume to improve the prognostic implications of the current TNM staging system, in which only the tumor size and the number of lymph node involvement are included.

Due to the retrospective design, some information was unavailable. A limitation of the study was the measurement technique of the nodal volume, in which only the CT or MRI scan imaging of the lymph node that contained the characteristics of possible metastasis were included. Therefore, the lymph node of size $<1 \mathrm{~cm}$, which may be metastatic, was excluded from the measure, which affected the total nodal volume. Due to the single-center study with limited number and distributions of the patients, further multi-center study with more number and distributions of the patients may provide more precise data and warrant the subgroup analysis, including comparing the nodal volume in the patients with the same $\mathrm{N}$ stage, to define the prognostic implications of the nodal volume and the $\mathrm{N}$ stage.

\section{Conclusions}

In patients with oral tongue cancer and cervical lymph node metastasis, the presence of a nodal volume of [?] $3.95 \mathrm{~cm}^{3}$ was a poor prognostic factor for distant metastasis. Therefore, the lymph node volume from the current imaging protocol may be useful in adjunct with the current staging system to predict the disease prognosis

\section{References}

(1) Tangjaturonrasme N, Vatanasapt P, Bychkov A. Epidemiology of head and neck cancer in Thailand. Asia-Pacific Journal of Clinical Oncology. 2017;14(1):16-22.

(2) Imsamran W, Chaiwerawattana A, Wiangnon S, Pongnikorn D, Suwanrungrung K, Sangrajrang S, et al. Cancer in Thailand: Vol. VIII, 2010-2012. Bangkok, Thailand: Ministry of Public Health, National Cancer Institute [and] Ministry of Education; 2015.

(3) Pruegsanusak K, Peeravut S, Leelamanit V, Sinkijcharoenchai W, Jongsatitpaiboon J, Phungrassami T, Chuchart K and Thongsuksai P, 2012. Survival and Prognostic Factors of Different Sites of Head and Neck Cancer: An Analysis from Thailand. Asian Pacific Journal of Cancer Prevention, 13(3), pp.885-890.

(4) Strongin A, Yovino S, Taylor R, Wolf J, Cullen K, Zimrin A et al. Primary Tumor Volume Is an Important Predictor of Clinical Outcomes Among Patients With Locally Advanced Squamous Cell Cancer of the Head and Neck Treated With Definitive Chemoradiotherapy. International Journal of Radiation Oncology, Biology, Physics. 2012;82(5):1823-1830.

(5) Nixon I, Palmer F, Lakin P, Kattan M, Lee N, Ganly I. Pathologically Determined Tumor Volume vs Pathologic T Stage in the Prediction of Outcome After Surgical Treatment of Oropharyngeal Squamous Cell Carcinoma. JAMA Otolaryngology-Head \& Neck Surgery. 2013;139(11):1151.

(6) Lin C, de Oliveira Santos A, Silva E, de Matos L, Moyses R, Kulcsar M et al. Tumor volume as an independent predictive factor of worse survival in patients with oral cavity squamous cell carcinoma. Head \& Neck. 2017;39(5):960-964.

(7) Amar A, Rapoport A, Curioni OA, Dedivitis RA, Cernea CR, Brandao LG. The density of metastatic lymph node as prognostic factor in squamous cell carcinoma of the tongue and floor of the mouth. Brazilian Journal of Otorhinolaryngology. 2012;78(3):86-90. 
(8) Chen C, Lin J, Chen K. Lymph node ratio as a prognostic factor in head and neck cancer patients. Radiation Oncology. 2015;10(1).

(9) Ganeshalingam S, Koh D. Nodal staging. Cancer Imaging. 2009;9(1).

(10) National Cancer Intelligence Network. Oral Cavity Cancer: recent survival trends [Internet]. United Kingdom: National Cancer Research Institute; 2013. Available from: https://www.cancerresearchuk.org/about-cancer/mouth-cancer/survival

\section{Figure legends}

Figure 1 - A: The measurement of lymph node volume (The yellow line indicates the border of the lymph node)

- B: Screenshot of the Eclipse application with the data of overall lymph node volume

Figure 2: A receiver operating characteristic (ROC) curve analysis of the optimal cut-off value to predict the mortality

Figure 3: The Kaplan-Meier curves and the log-rank test of the overall survival, disease-free survival, and metastasis-free survival

Table 1: Demographic data and clinical characteristics of the patients

Table 2: The median nodal volume of the patients

Table 3: Univariate and multivariate analysis of the factors associated with the survival outcomes 


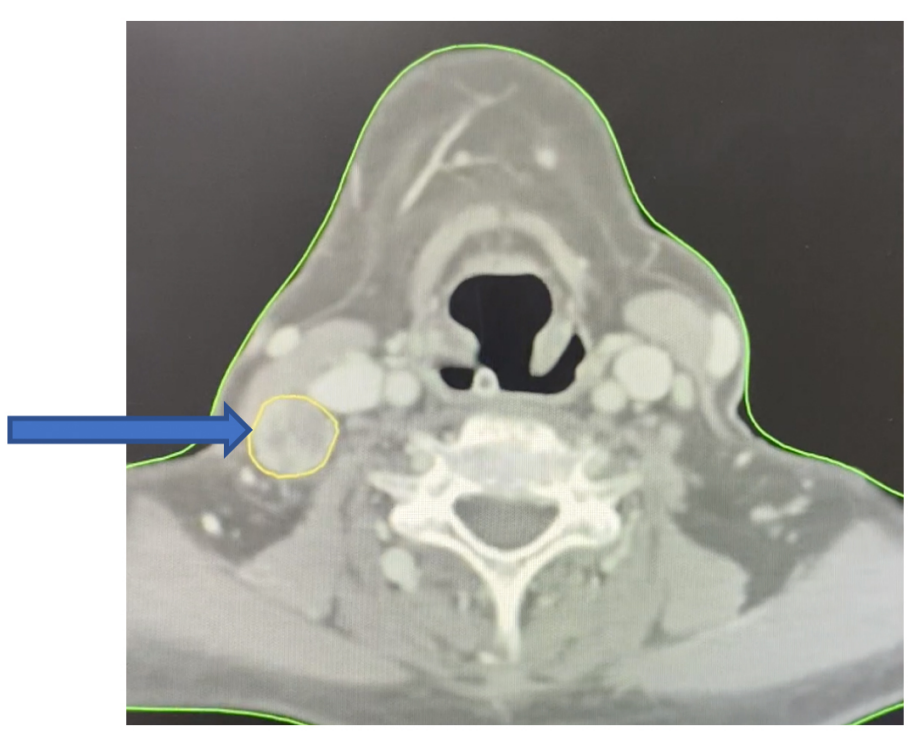

Figure1-A: The measurement of lymph node volume (The yellow line indicates the border of lymph node )

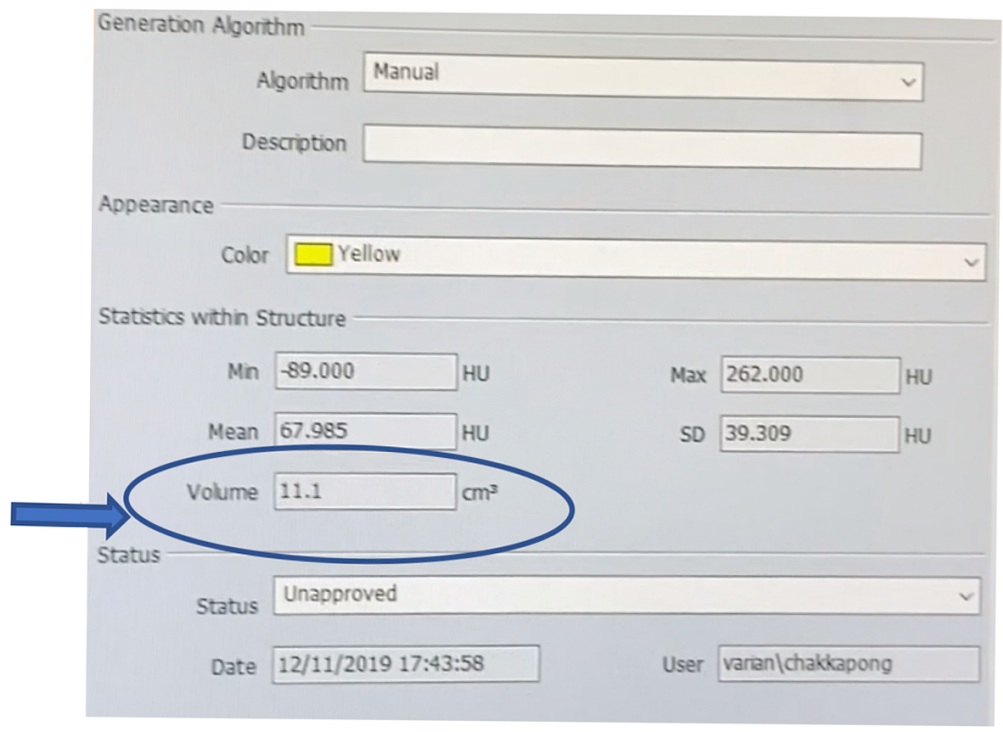

Figure1-B: Screenshot of the Eclipse application with the data of overall lymph node volume 


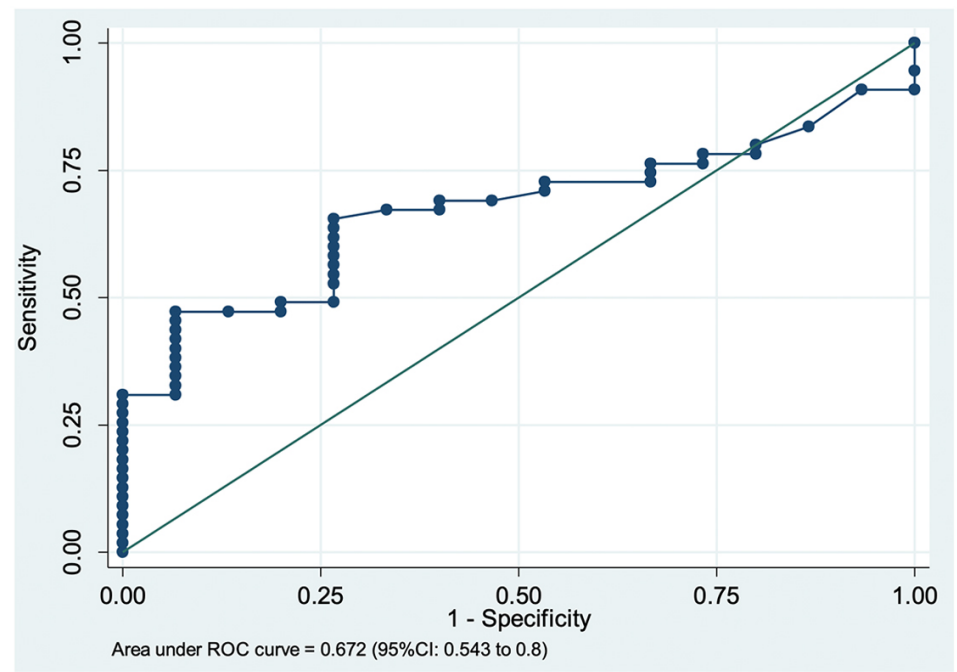

Figure2: A receiver operating charecteristics (ROC) curve analysis of the optimal cut-off value to predict the mortality

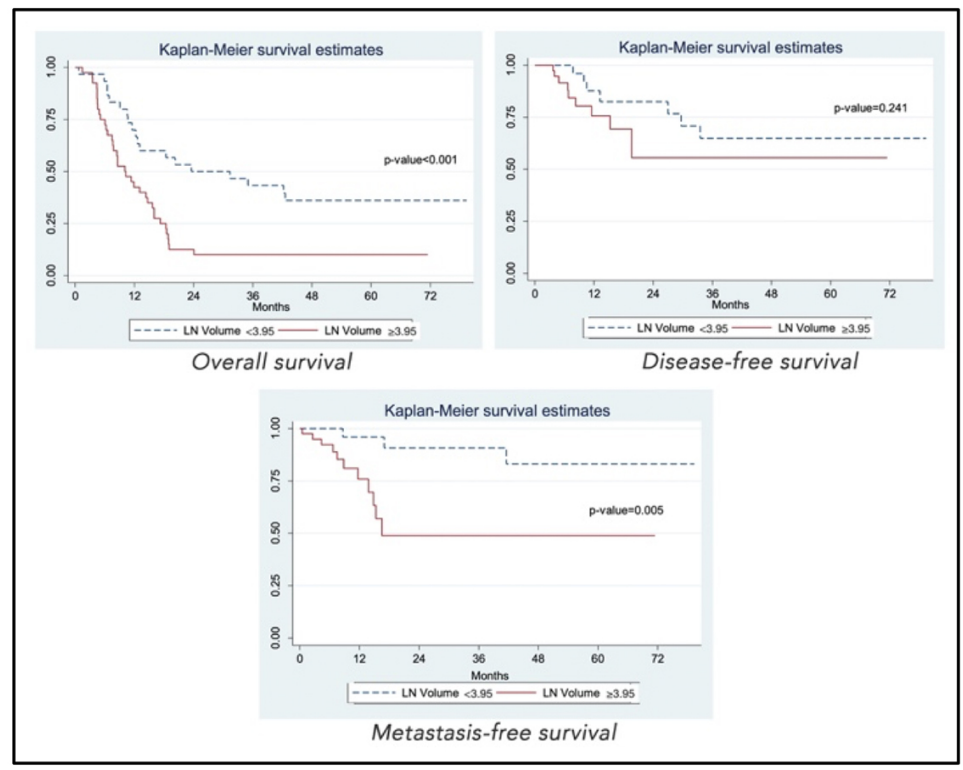

Figure3: The Kaplan-Meier curves and the log-rank test of the overall survival, disease-free survival, and metastasis-free survival

\section{Hosted file}

Table1.docx available at https://authorea.com/users/415852/articles/523619-nodal-volume-asa-prognostic-factor-in-oral-tongue-cancer-with-cervical-lymph-node-metastasis 


\section{Hosted file}

Table2.docx available at https://authorea.com/users/415852/articles/523619-nodal-volume-asa-prognostic-factor-in-oral-tongue-cancer-with-cervical-lymph-node-metastasis

\section{Hosted file}

Table 3.docx available at https://authorea.com/users/415852/articles/523619-nodal-volume-asa-prognostic-factor-in-oral-tongue-cancer-with-cervical-lymph-node-metastasis 